\title{
Bletilla striata polysaccharide has a protective effect on intestinal epithelial barrier disruption in TAA-induced cirrhotic rats
}

\author{
LEI LUO $^{1 *}$, ZHANG ZHOU $^{2 *}$, JUAN XUE $^{3 *}$, YAO WANG $^{4}$, JUAN ZHANG $^{5}$, \\ $\mathrm{XIN} \mathrm{CAI}^{6}$, YUQING $\mathrm{LIU}^{6}$ and $\mathrm{FAN} \mathrm{YANG}^{7}$ \\ ${ }^{1}$ Department of Gastroenterology, The Second People's Hospital of Yichang, Yichang, Hubei 443000; \\ ${ }^{2}$ Department of Anesthesia, Union Hospital, Tongji Medical College, Huazhong University of Science and Technology, \\ Wuhan, Hubei 430022; ${ }^{3}$ Department of Gastroenterology, Hubei Provincial Hospital of Traditional Chinese and \\ Western Medicine, Wuhan, Hubei 430015; ${ }^{4}$ Department of Infectious Diseases, Renmin Hospital of Wuhan University, \\ Wuhan, Hubei 430060; ${ }^{5}$ Department of Pulmonary Diseases, Jingmen City Hospital of Traditional Chinese Medicine, \\ Jingmen, Hubei 448000; ${ }^{6}$ School of Clinical Medical, Hubei University of Chinese Medicine, Wuhan, Hubei 430060; \\ ${ }^{7}$ Department of Hepatology, Hubei Provincial Hospital of Chinese Medicine, Wuhan, Hubei 430074, P.R. China
}

Received November 21, 2017; Accepted June 8, 2018

DOI: $10.3892 /$ etm.2018.6430

\begin{abstract}
It has been reported that intestinal epithelial barrier dysfunction serves an important role in the development of liver cirrhosis. However, at present there is no satisfactory treatment for intestinal mucosal lesions and ulcers associated with cirrhosis. The aim of the present study was to investigate the effect of Bletilla striata polysaccharide (BSP) on intestinal epithelial barrier disruption in rats with thioacetamide (TAA)-induced liver cirrhosis. Rats were randomly divided into 5 groups $(\mathrm{n}=10)$ : BSP low dosage $(15 \mathrm{mg} / \mathrm{kg})$, BSP middle dosage $(30 \mathrm{mg} / \mathrm{kg})$, BSP high dosage $(60 \mathrm{mg} / \mathrm{kg})$, experiment and control groups. All groups except control group were administered with TAA (200 mg/kg/day) to induce liver cirrhosis. Following modeling, rats in the low, middle and high-dose BSP groups received BSP. ELISA kits were used to measure the endotoxin, alanine transaminase (ALT) and aspartate transaminase (AST) levels in the portal vein, while interleukin (IL)-6 and tumor necrosis factor (TNF)- $\alpha$ expression in the ileal tissue was measured. Reverse transcription-quantitative polymerase chain reaction (RT-qPCR) and western blotting were used to detect the expression of zonula occludens (ZO)-1 and occludin mRNA and protein, respectively. Intestinal epithelial tissue pathology was detected using hematoxylin-eosin (HE) staining. Immunohistochemistry was performed to assess the
\end{abstract}

Correspondence to: Professor Fan Yang, Department of Hepatology, Hubei Provincial Hospital of Chinese Medicine, 856 Luoyu Road, Hongshan, Wuhan, Hubei 430074, P.R. China E-mail: yangfan@hbhtcm.com

*Contributed equally

Key words: liver cirrhosis, Bletilla striata polysaccharide, intestinal barrier, tight junction, endotoxin expression of ZO-1 and occludin in intestinal epithelial tissues. Following treatment with BSP, ALT, AST and endotoxin levels in the portal vein, as well as IL- 6 and TNF- $\alpha$ expression in ileal tissues, were significantly decreased compared with model group $(\mathrm{P}<0.05$ or 0.01$)$. Furthermore, BSP treatment upregulated the expression of ZO-1 and occludin mRNA and protein compared with the model group $(\mathrm{P}<0.05$ or 0.01$)$ and cytoplasmic staining for these proteins was increased. The degree of intestinal epithelial tissue pathological damage was significantly reduced in the BSP groups. In conclusion, BSP is able to reduce endotoxin levels, inhibit the inflammatory cytokines IL-6 and TNF- $\alpha$ and elevate expression of ZO-1 and occludin at tight junctions. Together, these results suggest a novel protective agent for the restoration of intestinal epithelial barrier disruption.

\section{Introduction}

Cirrhosis is the end stage of chronic liver disease and a major risk factor of hepatocellular carcinoma (1). In recent years, it has been reported that intestinal barrier disruption and increased intestinal permeability may lead to bacterial translocation (BT) and absorption of endotoxemia, which increases susceptibility to infection (2). The intestinal barrier includes secreted mucus and the epithelial cell layer itself. Increased intestinal permeability is the main cause of intestinal barrier dysfunction (3). Intestinal epithelial monolayer cells are the main barrier of the intestinal mucosa, which serves an important role in preventing the infiltration of a number of harmful substances, allergens, toxins and luminal microbial pathogens $(4,5)$. The functional integrity of the epithelial barrier is maintained by intercellular tight junctions (TJs), which include the transmembrane protein occludin, claudins and the cytoplasmic protein zonula occludens 1 (ZO-1) (6). Intercellular TJ protein destruction is associated with a number of diseases, including cirrhosis (7). Therefore, restoring the integrity of the intestinal barrier is an important step in preventing intestinal endotoxemia. 
Lipopolysaccharide (LPS), also known as endotoxin, is the main component of the outer wall of Gram-negative bacteria and is released from the bacterial cell wall via shedding or bacterial lysis $(8,9)$. Endotoxin causes an increase in intestinal permeability by activating proinflammatory cytokines, including tumor necrosis factor- $\alpha(\mathrm{TNF}-\alpha)$, interleukin-6 (IL-6) and nitric oxide, and injuring the intercellular TJ between epithelial cells $(10,11)$. It has been reported that patients with cirrhosis develop intestinal endotoxemia with an incidence of 79-92\% (12), while other clinical and experimental studies suggest that liver cirrhosis is associated with endotoxemia $(13,14)$.

Bletilla striata is a traditional medicine that has been widely used to treat ulcers, bleeding, burns, bruises and many other diseases for $>1,000$ years $(15,16)$. B. striata polysaccharide (BSP) is extracted from $B$. striata and consists of $\alpha$-mannose, $\beta$-mannose and $\beta$-glucose (17). BSP has a number of biological functions, including anti-inflammatory (18), anti-tumor (19), anti-fibrosis (20) and antibacterial (21) properties, as well as inducing endothelial cell proliferation and vascular endothelial growth-factor expression (16). However, to the best of our knowledge, no previous studies have investigated the protective role of BSP in epithelial barrier disruption.

The aim of the present study was to investigate whether BSP is able to restore the integrity and function of the intestinal epithelial barrier via inhibiting the expression of inflammatory cytokines and improving TJs in the small intestine of rats with thioacetamide (TAA)-induced liver cirrhosis.

\section{Materials and methods}

Chemicals and reagents. BSP ( $295 \%$ titration) was purchased from Dalian MeiLun Biotechnology Co., Ltd. (Dalian, China). TAA was purchased from Sigma-Aldrich (Merck KGaA, Darmstadt, Germany) and dissolved in physiological saline $(0.9 \% \mathrm{NaCl})$ to give a $4 \%$ solution for animal experiments. Rabbit anti-rat zonula occludens (ZO)-1 and occludin were obtained from ProteinTech Group, Inc. (Chicago, IL, USA). ELISA kits for serum alanine aminotransferase (ALT; cat. no. C009-2) and aspartate aminotransferase (AST; cat. no. C0010-2) were purchased from Nanjing Jiancheng Bioengineering Institute (Nanjing, China). ELISA kits for IL-6 (cat. no. E-EL-R0015), TNF- $\alpha$ (cat. no. E-EL-R0019) and endotoxin (cat. no. E-EL-R0589) were purchased from Elabscience Biotechnology (Wuhan, China). GAPDH and horseradish peroxidase (HRP)-labeled secondary antibodies were acquired from Wuhan Boster Biotechnology Co., Ltd. (Wuhan, China). RNAiso Plus, PrimeScript ${ }^{\mathrm{TM}}$ RT reagent and SYBR Premix Ex Taq kits were purchased from Takara Bio, Inc. (Otsu, Japan).

Animals and experimental protocols. A total of 50 healthy male Sprague-Dawley rats (age, 6 weeks; weight, 180-220 g) were purchased from Hubei Provincial Center for Disease Control and Prevention (Wuhan, China). All animals were housed at $22-25^{\circ} \mathrm{C}$, relative humidity of $50 \pm 10 \%$ with a $12 \mathrm{~h}$ light/dark cycle and free access to water and food. All animal experiment protocols used in the present study followed internationally accepted principles and were approved by The Institutional Animal Care and Use Committee of Tongji Medical College, Huazhong University of Science and Technology (Wuhan,
China). Rats were randomly divided into 5 groups $(n=10)$ as follows: $15 \mathrm{mg} / \mathrm{kg}$ BSP, $30 \mathrm{mg} / \mathrm{kg}$ BSP, $60 \mathrm{mg} / \mathrm{kg}$ BSP, experiment and control groups. Rats in the BSP and experimental groups were administered intraperitoneally (IP) with TAA (200 mg/kg, dissolved in $0.9 \% \mathrm{w} / \mathrm{v}$ saline solution to give $4 \%$ solution) twice per week for 14 weeks to induce liver cirrhosis. The control group received IP injections of the same volume of saline $(0.9 \% \mathrm{w} / \mathrm{v})$. At 12 weeks, 3 rats from the experimental and control groups were selected at random and anesthetized by IP. Injection with $10 \%$ chloral hydrate $(300 \mathrm{mg} / \mathrm{kg})$. The abdominal cavity was opened with scissors. Livers were harvested and examined using hematoxylin and eosin (HE) staining to confirm the development of liver cirrhosis. Rats in the experimental group were administered with saline at $1 \mathrm{ml} / 100 \mathrm{~g}$ once daily by gavage for 2 weeks while rats in the BSP groups were administered with BSP at 15,30 or $60 \mathrm{mg} / \mathrm{kg}$ for 2 weeks at concentrations of $1.5,3$ or $6 \mathrm{mg} / \mathrm{ml}$ at $1 \mathrm{ml} / 100 \mathrm{~g}$ once daily by gavage. TAA administration was continued until the end of the 14-week experimental period. At 14 weeks, the remaining rats were injected IP with $10 \%$ chloral hydrate $(300 \mathrm{mg} / \mathrm{kg}$ ) and dissected. Blood samples were collected from portal veins and intestinal tissues were harvested for further analysis.

Serum alanine aminotransferase (ALT) and aspartate aminotransferase (AST) analysis. Blood samples were obtained and centrifuged at $1,760 \mathrm{xg}$ for $15 \mathrm{~min}$ at $4^{\circ} \mathrm{C}$ to collect the serum. Serum ALT and AST levels were determined using assay kits according to the manufacturer's protocols.

Measurement of serum endotoxin levels. Endotoxin levels were measured in all groups using specific ELISA kit according the manufacturer's instructions. Briefly, portal vein blood samples were collected and centrifuged at $1,760 \mathrm{x}$ g for $15 \mathrm{~min}$ at $4^{\circ} \mathrm{C}$ to collect plasma, which was subsequently diluted with endotoxin-free water and incubated at $70^{\circ} \mathrm{C}$ for $10 \mathrm{~min}$. Samples were then incubated in ice-cold water for $3 \mathrm{~min}$, following which limulus reagents and $200 \mu \mathrm{l}$ processed mixing solution were added and $100 \mu \mathrm{l}$ of mixture was transferred to a 96-well microplate for analysis. The concentration of endotoxin was calculated using a standard curve and the absorbance of each well was measured at $450 \mathrm{~nm}$ using a microplate reader to calculate the concentration of endotoxin $(\mathrm{EU} / \mathrm{ml})$.

ELISA measurement of IL- 6 and TNF- $\alpha$ in ileal tissues. Harvested ileal tissues were cut into small pieces and homogenized in pre-cooled PBS containing proteinase inhibitors (BioSharp, Hefei, China). The supernatant of the homogenate was collected and ELISA was performed to measure the concentrations of IL- 6 and TNF- $\alpha$ according to the manufacturer's protocol. All samples were tested in duplicate.

Reverse transcription-quantitative polymerase chain reaction $(R T-q P C R)$. Total RNA was extracted from rat ileal tissues using RNAiso Plus according to the manufacturer's protocol and stored at $-80^{\circ} \mathrm{C}$ prior to use. cDNA was produced using a PrimeScript $^{\mathrm{TM}} \mathrm{RT}$ reagent kit with the following temperature protocol: $37^{\circ} \mathrm{C}$ for $15 \mathrm{~min}$ and $85^{\circ} \mathrm{C}$ for 5 sec. qPCR reactions were performed using a StepOne Plus device (Applied Biosystems; Thermo Fisher Scientific, Inc., Waltham, MA, 


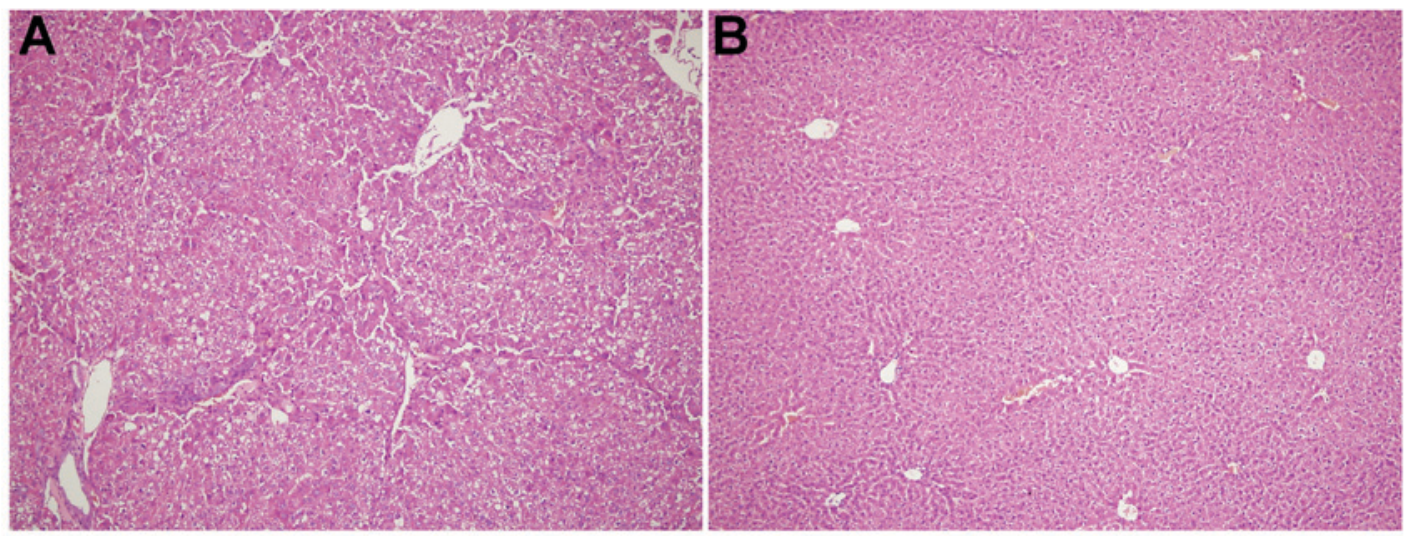

Figure 1. Hematoxylin and eosin stained liver tissues from the (A) experiment and (B) control groups following thioacetamide administration for 12 weeks. Magnification, $\mathrm{x} 100$

USA) at $95^{\circ} \mathrm{C}$ for $10 \mathrm{sec}$, followed by 40 cycles of $95^{\circ} \mathrm{C}$ for $5 \mathrm{sec}$ and $60^{\circ} \mathrm{C}$ for $20 \mathrm{sec}$ according to the instructions of the SYBR Premix Ex Taq kit. Data were quantified using the $2^{-\Delta \Delta C q}$ method (22). Primers were synthesized by GenScript (Piscataway, NJ, USA) and sequences were as follows: ZO-1, forward 5'-GCTCACCAGGGTCAAAATGT-3' and reverse 5'-GGCTTAAAGCTGGCAGTGTC-3'; occludin, forward 5'-TTACGGCTATGGAGGGTACAC-3' and reverse 5'-GAC GCTGGTAACAAAGATCAC-3'; and GAPDH, forward 5'-GGAAAGCTGTGGCGTGAT-3' and reverse 5'-AAGGTG GAAGAATGGGAGTT-3'.

Western blotting. Western blotting was performed as previously described (23). Briefly, total proteins were extracted from the ileum tissue using radioimmunoprecipitation assay buffer (Beyotime Institute of Biotechnology, Wuhan, China). A bicinchoninic protein assay kit was used to measure protein concentrations. A total of $20 \mu \mathrm{g}$ proteins in each sample were separated on $10 \%$ SDS-PAGE gels and transferred to polyvinylidene fluoride membranes. Membranes were blocked with PBS containing 5\% nonfat milk powder for $1 \mathrm{~h}$ at room temperature and incubated overnight at $4^{\circ} \mathrm{C}$ with antibodies against ZO-1 (1:1,000; cat. no. 21773-1-AP), occludin (1:1,000; cat. no. 13409-1-AP) and GAPDH (1:5,000; cat. no. BM1985). Membranes were washed three times with TBST and incubated at $4^{\circ} \mathrm{C}$ with HRP-labeled secondary antibody $(1: 2,000$; cat. no. BA1058) for $1 \mathrm{~h}$. Membranes were washed with TBST three times and enhanced chemiluminescence (Millipore; Merck KGaA, Darmstadt, Germany) was used to identify immunoreactive bands and followed the manufacturer's protocol. Bands underwent densitometric analysis using the Fuji ultrasonic-Doppler velocity profile (UVP) system and Image J software (v.1.50; National Institutes of Health, Bethesda, MD, USA).

Histopathology. Terminal ileal samples were collected, fixed in $4 \%$ paraformaldehyde solution at room temperature for $24 \mathrm{~h}$, embedded in paraffin and cut into 4-5 $\mu \mathrm{m}$ sections. Sections were deparaffinized using dimethyl benzene, dehydrated using alcohol for 2-5 min at room temperature, washed with distilled water and stained with hematoxylin at room temperature for 10-30 min. Excess stain was removed and sections were incubated with $1 \%$ acid alcohol for $30 \mathrm{sec}$ at room temperature and dehydrated using gradient alcohol (70, 85, 95 and 100\%; 2-3 min, respectively) at room temperature. Sections were stained with $0.5 \%$ eosin at room temperature for $2-5 \mathrm{~min}$, incubated with $95 \%$ alcohol at room temperature for $2 \mathrm{~min}$ and with xylene at room temperature for $10 \mathrm{~min}$ and finally sealed with a neutral balsam. Sections were mounted and histological changes were observed using light microscopy (magnification, $\mathrm{x} 200)$.

Immunohistochemistry (IHC) of ileal proteins. The ileal tissue specimens were cut into $10 \mu \mathrm{m}$ sections following dewaxing and hydrating. Sections were treated with $3 \% \mathrm{H}_{2} \mathrm{O}_{2}$-methanol to block endogenous peroxidase activity, following which they were incubated with 5\% normal goat serum (Wuhan Boster Biological Technology,Ltd.) at room temperature for $10 \mathrm{~min}$ and incubated with ZO-1 and occludin antibodies (dilution 1:200) overnight at $4^{\circ} \mathrm{C}$. Slides were subsequently washed with PBS and incubated with a biotinylated secondary antibody (1:500; cat. no. SA00004-2; Wuhan Boster Biological Technology, Ltd.) for $1 \mathrm{~h}$ at room temperature. Slides were washed with PBS again and incubated with HRP-labeled streptavidin (1:200; ab214880; Abcam) at $37^{\circ} \mathrm{C}$ for $1 \mathrm{~h}$. Samples were developed using diaminobenzene (DAB) at room temperature for $30 \mathrm{sec}$ and counterstained with hematoxylin at room temperature for $5 \mathrm{~min}$. Slides were rinsed in distilled water and dehydrated, following which they were observed under a light microscope (magnification, x100).

Statistical analysis. Data are presented as the mean \pm standard error of the mean. Data were compared between groups using one-way analysis of variance with the Student-Newman-Keuls post-hoc test. All statistical analyses were performed with SPSS software version 12.0 (SPSS, Inc., Chicago, IL, USA). P<0.05 was considered statistically significant difference. Graphs were created using GraphPad Prism software (version 6; GraphPad Software, Inc., La Jolla, CA, USA).

\section{Results}

Liver histological changes in rats following treatment with thioacetamide for 12 weeks. As shown in Fig. 1A, the liver of 
A

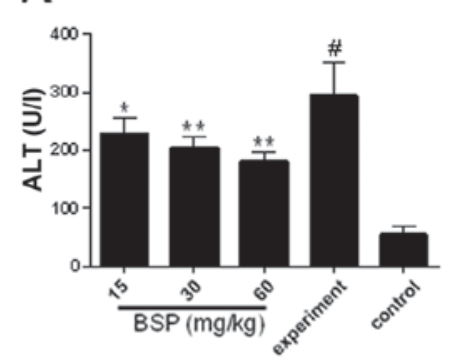

B

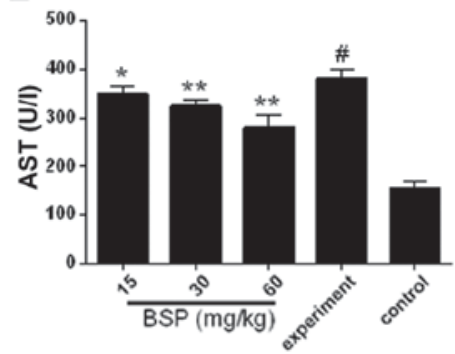

C

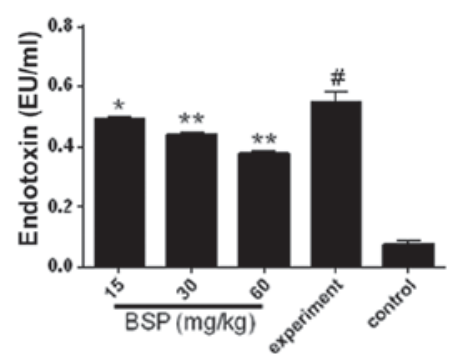

Figure 2. ELISA was used to assess the effect of BSP on serum (A) ALT, (B) AST and (C) endotoxin levels. ${ }^{*} \mathrm{P}<0.05$ and ${ }^{* *} \mathrm{P}<0.01$ vs. experiment group; ${ }^{*} \mathrm{P}<0.01$ vs. control group. BSP, Bletilla striata polysaccharide; ALT, alanine transaminase; AST, aspartate transaminase.

A

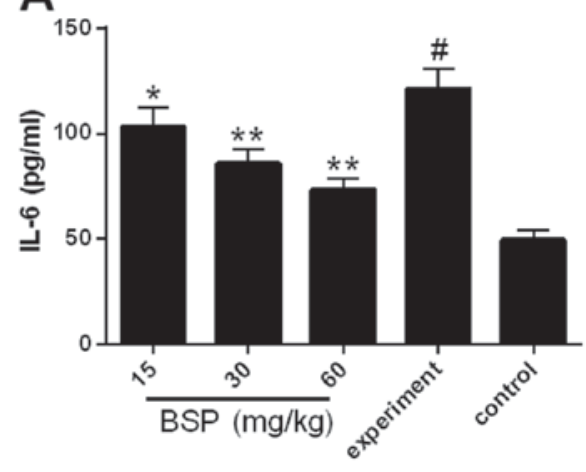

B

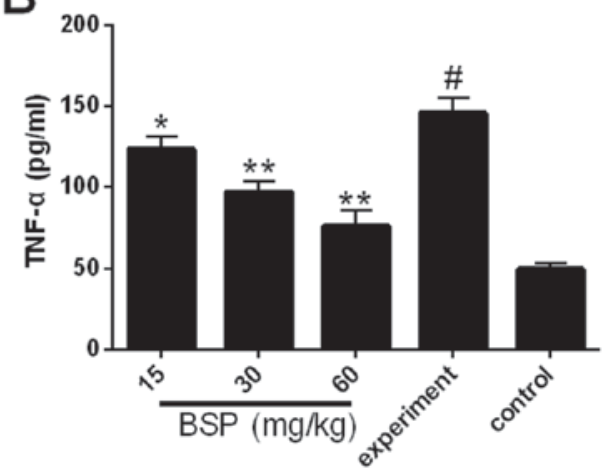

Figure 3. Effects of BSP on expression of (A) IL-6 and (B) TNF- $\alpha$ in rat ileal tissues. ${ }^{*} \mathrm{P}<0.05$ and ${ }^{* *} \mathrm{P}<0.01$ vs. experiment group; ${ }^{*} \mathrm{P}<0.01$ vs. control group. Data are presented as the mean \pm standard error of the mean $(\mathrm{n}=3)$. BSP, Bletilla striata polysaccharide; IL, interleukin; TNF, tumor necrosis factor.
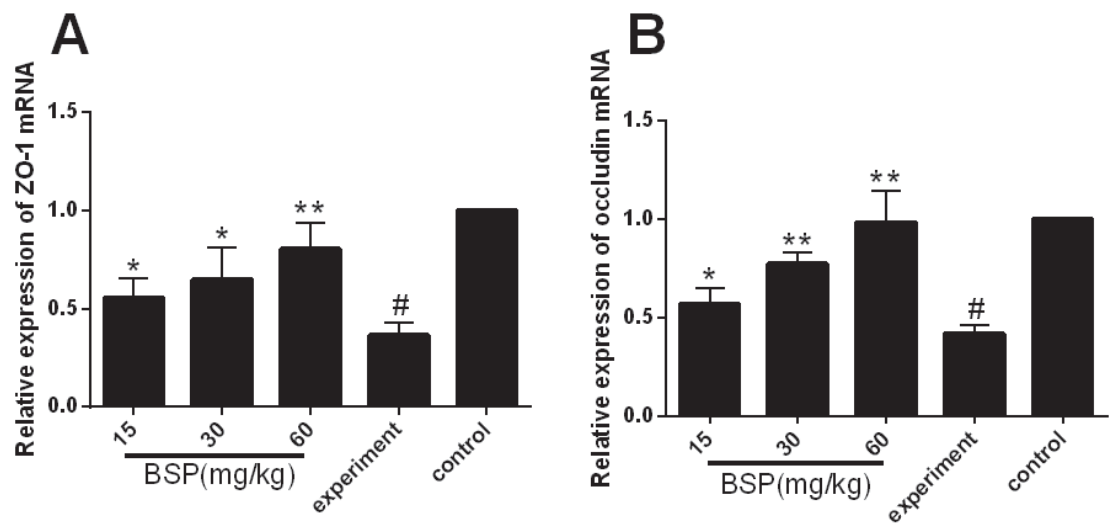

Figure 4. Effects of BSP on the expression of (A) ZO-1 and (B) occludin mRNA in rat ileal tissues. ${ }^{~} \mathrm{P}<0.05$ and ${ }^{* *} \mathrm{P}<0.01$ vs. experiment group; ${ }^{*} \mathrm{P}<0.01$ vs. control group. Data are presented as the mean \pm standard error of the mean. BSP, Bletilla striata polysaccharide; ZO, Zonula occludens.

the experiment group exhibited a lobular structure destruction, inflammatory cell infiltration and the loss of structural integrity. The liver of the control group (Fig. 1B) revealed structural liver integrity without inflammatory cell infiltration.

Activity of transaminases. The expression of serum AST and ALT was assessed using ELISA kits and was demonstrated to be significantly increased in the experiment group compared with the control (Fig. 2A and B). However, treatment with BSP at all concentrations resulted in a significant reduction in serum
ALT and AST expression compared with the experiment group (Fig. 2A and B).

$B S P$ reduces TAA-induced plasma endotoxin level. As shown in Fig. 2C, plasma endotoxin levels were significantly increased in the experiment group compared with the control group. Treatment with BSP at all dosages obviously decreased endotoxin levels compared with the experiment group.

Effects of BSP on inflammatory cytokines expression in ileal tissue. Levels of the inflammatory cytokines IL-6 and TNF- $\alpha$ 
A

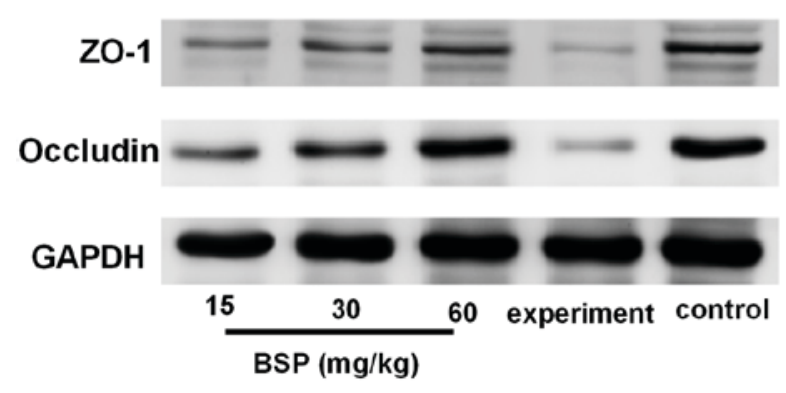

B

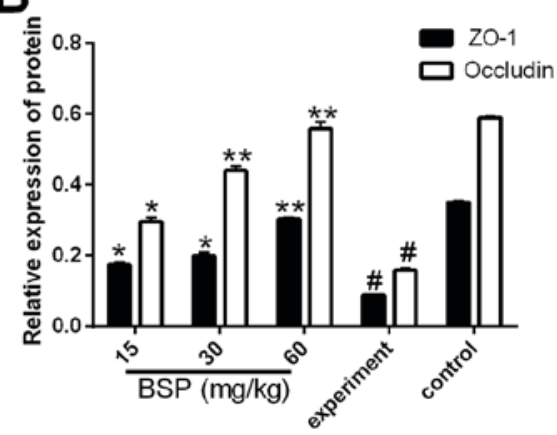

Figure 5. ZO-1 and occludin expression in rat ileal tissues were (A) measured using western blotting and (B) quantified using GAPDH as a reference following BSP treatment. ${ }^{*} \mathrm{P}<0.05$ and ${ }^{* *} \mathrm{P}<0.01$ vs. experiment group; ${ }^{\#} \mathrm{P}<0.01$ vs. control group. Data are presented as the mean \pm standard error of the mean. ZO, Zonula occludens; BSP, Bletilla striata polysaccharide.
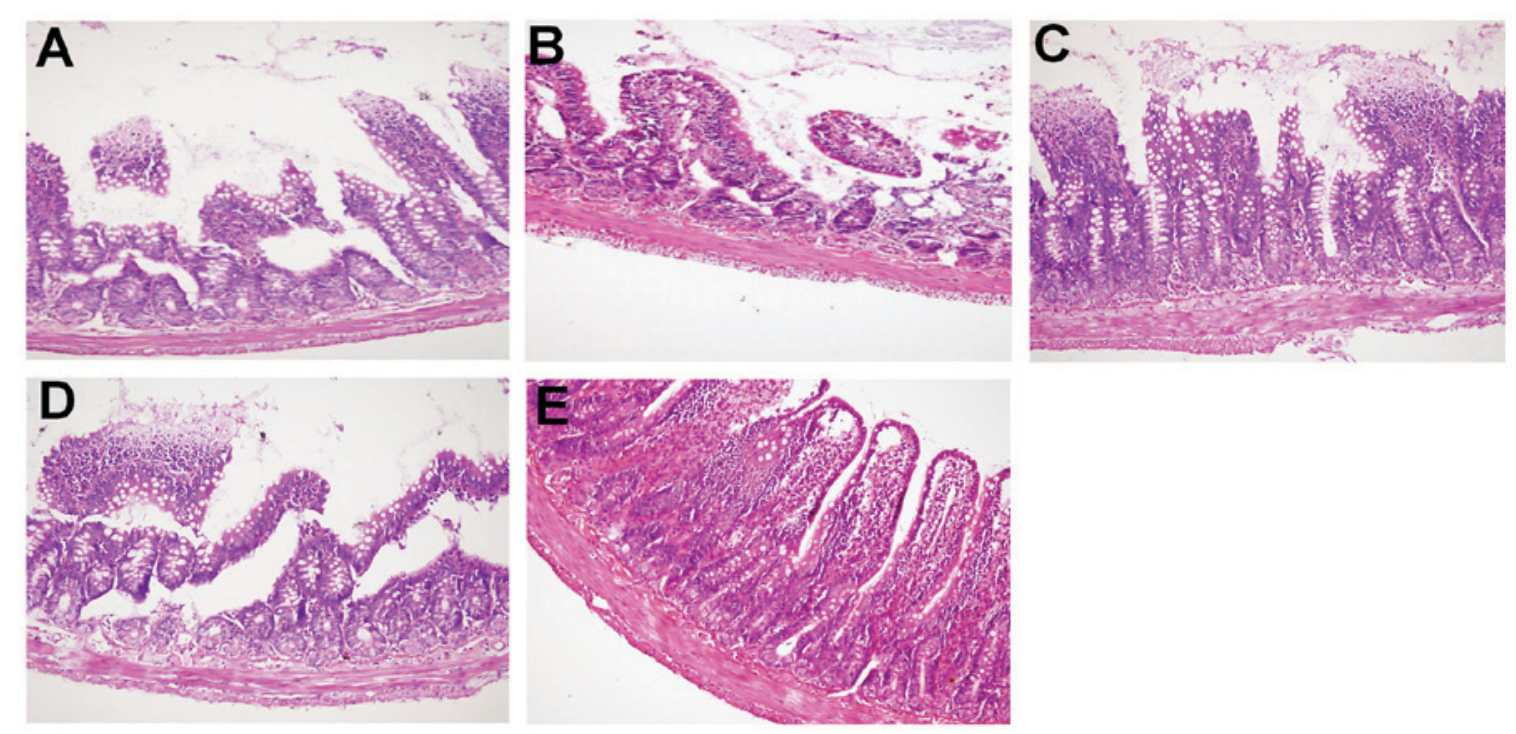

Figure 6. Pathological changes in ileal tissues following treatment with BSP were assessed using hematoxylin and eosin staining (magnification, $\mathrm{x} 200$ ). Representative images of (A) 15, (B) 30 and (C) $60 \mathrm{mg} / \mathrm{kg}$ BSP groups, (D) experiment group and (E) control group. BSP, Bletilla striata polysaccharide.

were measured in the ileal tissues. Compared with the control group, the expression of IL- 6 and TNF- $\alpha$ was significantly increased in the experiment group (Fig. 3). However, following treatment with BSP at all concentrations, IL-6 and TNF- $\alpha$ expression was significantly decreased compared with the experiment group (Fig. 3).

Effects of BSP on the expression of ZO-1 and occludin $m R N A$ in ileal tissues. RT-qPCR was used to assess the effect of BSP on ZO-1 and occludin mRNA expression. It was demonstrated that ZO-1 and occludin mRNA was downregulated in the experiment group compared with the control group (Fig. 4), while treatment with BSP significantly upregulated ZO-1 and occludin mRNA compared with the experiment group (Fig. 4).

Effects of BSP on the expression of ZO-1 and occludin protein in ileal tissues. Compared with the control group, the expression of ZO-1 and occludin proteins in the experiment group was significantly decreased (Fig. 5). However, following treatment with BSP at different concentrations, the expression of ZO-1 and occludin proteins was significantly increased compared with the experiment group (Fig. 5).

Histological changes in ileal tissues. Intestinal mucosal damage was ameliorated in the BSP treatment groups, with less intestinal wall structure destruction and more villi observed (Fig. 6A-C). These effects were most notable in the $60 \mathrm{mg} / \mathrm{kg}$ BSP group (Fig. 6C). In the experiment group (Fig. 6D), the structure of the intestinal wall was damaged and the intestinal villus was fractured, shortened and atrophic. Histopathology of the intestinal mucosa in the control group revealed that the intestinal layer had a clear structure with intact surface epithelium and neatly arranged intestinal villus (Fig. 6E).

Effect of BSP on ZO-1 and occludin protein expression in intestinal tissues. IHC was performed and the results demonstrated that the control group was positively stained for ZO-1 and occludin, with dark brown coloration (Fig. 7A). In the experiment group, however, positive ZO-1 and occludin staining was significantly decreased (Fig. 7A and B). The degree of positive staining observed in the BSP treatment groups was 


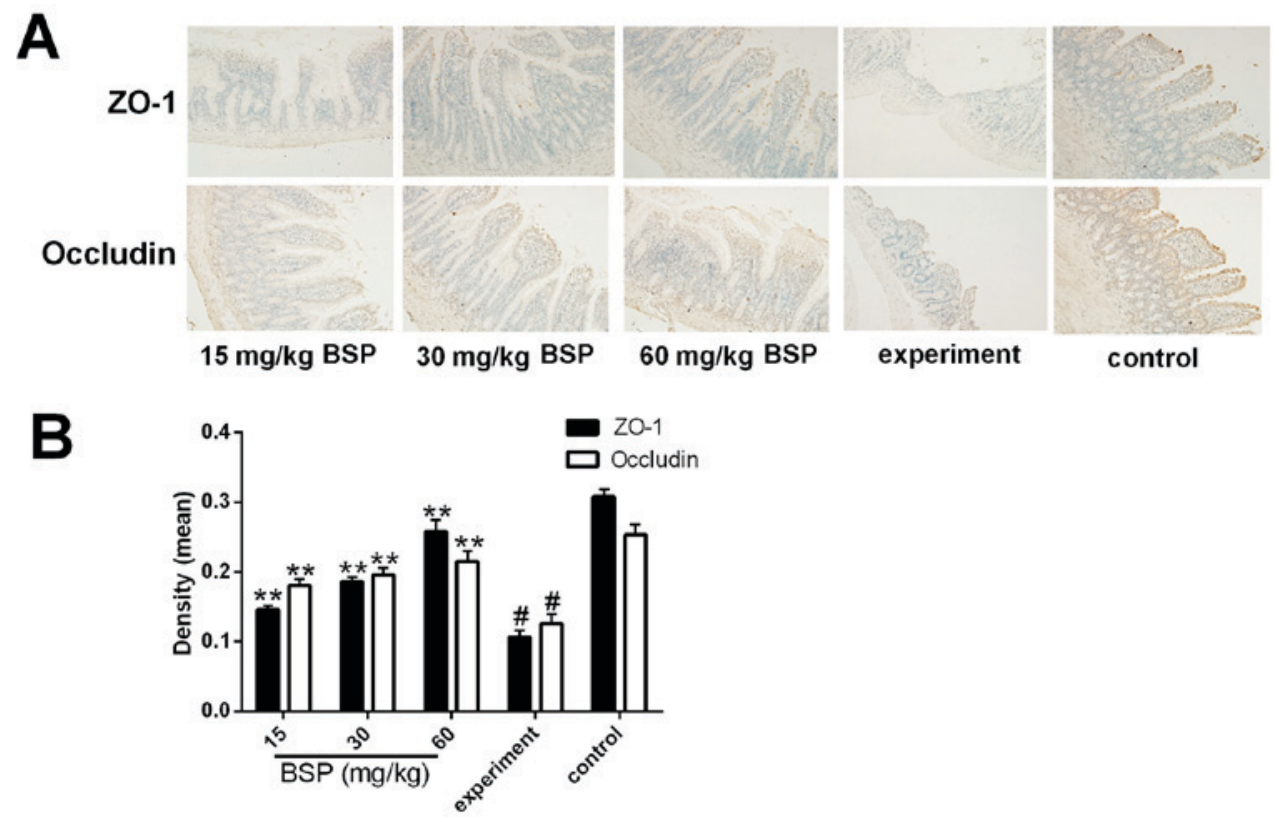

Figure 7. Immunohistochemistry was used to assess the effects of BSP on ZO-1 and occludin expression (magnification, $x 100$ ). (A) Representative images of tissues from each group and (B) quantified results. ${ }^{* *} \mathrm{P}<0.01$ vs. experiment group; ${ }^{*} \mathrm{P}<0.01$ vs. control group. Data are presented as the mean \pm standard error of the mean. BSP, Bletilla striata polysaccharide; ZO, Zonula occludens.

significantly increased in compared with the experiment group (Fig. 7A and B).

\section{Discussion}

Cirrhosis is a common chronic progressive liver disease, which is caused by one or more long-term or repeated effects, including chronic viral infection (hepatitis B and C), immunologic attack and parasitic diseases (24). The significance of intestinal epithelial barrier dysfunction as a critical cofactor in liver cirrhosis has been reported over the past decades $(25,26)$. Intestinal epithelial cells prevent the invasion of unwelcome microorganisms, while LPS-induced intestinal barrier dysfunction leads to an increase in intestinal mucosal permeability, which accelerates the release of inflammatory factors (27) and intestinal cytokines, resulting in expedited intestinal damage and inflammation (28). IL-6 and TNF- $\alpha$ are the critical cytokines associated with inflammation and are common treatment targets for inflammatory diseases, including liver cirrhosis $(29,30)$. TNF- $\alpha$ serves an important role in the progression of intestinal epithelial barrier injury (31) and is able to initiate the production of cytokines, including IL-6, which further exacerbates intestinal epithelial barrier damage (32). A clinical study reported that TNF- $\alpha$ and IL-6 were significantly upregulated in patients with cirrhosis (33). Taken together, these results suggest that inhibiting the expression of inflammatory factors may be an effective method for protecting against intestinal endotoxemia.

In the present study, intestinal IL- 6 and TNF- $\alpha$ were upregulated in a TAA-induced cirrhosis rat model and this effect was inhibited by BSP treatment. These results suggest that cirrhosis is associated with the overexpression of inflammatory cytokines and that BSP is able to effectively ameliorate this.

TJs are localized at the apical end of lateral membranes and are some of the most important structures responsible for preventing toxic substances from the intestine penetrating the surrounding tissue through the epithelial cell layer $(34,35)$. $\mathrm{TJ}$ proteins are located between epithelial cells and comprise a variety of transmembrane and membrane-associated proteins (36), including ZO-1 and occludin. The primary function of these molecules is to act as a selective intercellular barrier to regulate the diffusion of macromolecules (37). Occludin is a four-transmembrane protein, comprising four putative membrane-spanning segments (38), which is exclusively localized at TJs in epithelial and endothelial cells (39). It has been reported that high occludin expression is correlated with low endothelium permeability (40). Another study demonstrated that downregulating the expression of occludin results in increased intestinal permeability, allowing bacteria, endotoxins and other macromolecules to enter the body (41). As such, measuring occludin levels may reflect the integrity of the intestinal barrier (42-44). ZO-1 was the first TJ protein to be discovered (45) and is directly associated with occludin, serving an important role in regulating TJ barrier function (46). It has been reported that LPS is able to decrease the expression of ZO-1 and occludin (47), which is consistent with the results of the present study.

In the present study, it was demonstrated that BSP is able to upregulate the expression of ZO-1 and occludin in a TAA-induced cirrhosis rat model. BSP is also able to reduce endotoxin levels and decrease the degree of intestinal mucosa damage. Taken together, these results suggest that BSP is able to preserve the barrier function of the epithelium by keeping TJs intact.

In summary, the results of the present study indicate that BSP is able to protect against intestinal epithelial barrier disruption in a TAA-induced liver cirrhosis rat model via reducing endotoxin levels, inhibiting inflammatory cytokine expression and improving TJs, suggesting that BSP may have potential as a treatment for intestinal endotoxemia. However, it is important to elucidate the underlying mechanism of BSP in intestinal 
endotoxemia. Future studies on the intestinal endotoxemia mechanisms of BSP may give a more complete understanding and further the development of novel methods to prevent and treat intestinal endotoxemia.

\section{Acknowledgements}

Not applicable.

\section{Funding}

The present study was supported by The Second Batch of Young Middle-aged Talents in Wuhan.

\section{Availability of data and materials}

The datasets used and/or analyzed during the current study are available from the corresponding author on reasonable request.

\section{Authors' contributions}

FY and YW designed and directed the experiment. LL, XC and YL performed the experiments. ZZ, JX and JZ performed the statistical analysis. LL wrote the manuscript. All authors read and approved the final manuscript.

\section{Ethics approval and consent to participate}

The study was approved by The Institutional Animal Care and Use Committee of Tongji Medical College, Huazhong University of Science and Technology (Wuhan, China). All animal experiment protocols used in the present study followed internationally accepted principles.

\section{Patient consent for publication}

Not applicable.

\section{Competing interests}

The authors have no potential competing interests to disclose.

\section{References}

1. Gogoi-Tiwari J, Köhn-Gaone J, Giles C, Schmidt-Arras D, Gratte ED, Elsegood CL, McCaughan GW, Ramm GA, Olynyk JK and Tirnitz-Parker JEE: The murine choline-deficient, ethionine-supplemented (CDE) diet model of chronic liver injury. J Vis Exp: Oct 21, 2017.

2. Wang H, Zhang W, Zuo L, Zhu W, Wang B, Li Q and Li J: Bifidobacteria may be beneficial to intestinal microbiota and reduction of bacterial translocation in mice following ischaemia and reperfusion injury. Br J Nutr 109: 1990-1998, 2013.

3. Arrieta MC, Bistritz L and Meddings JB: Alterations in intestinal permeability. Gut 55: 1512-1520, 2006.

4. Groschwitz KR and Hogan SP: Intestinal barrier function: Molecular regulation and disease pathogenesis. J Allergy Clin Immunol 124: 3-20, 2009.

5. Turner JR: Intestinal mucosal barrier function in health and disease. Nat Rev Immunol 9: 799-809, 2009.

6. Clark JA, Doelle SM, Halpern MD, Saunders TA, Holubec H, Dvorak K, Boitano SA and Dvorak B: Intestinal barrier failure during experimental necrotizing enterocolitis: Protective effect of EGF treatment. Am J Physiol Gastrointest Liver Physiol 291: G938-G949, 2006.
7. Pijls KE, Koek GH, Elamin EE, de Vries H, Masclee AA and Jonkers DM: Large intestine permeability is increased in patients with compensated liver cirrhosis. Am J Physiol Gastrointest Liver Physiol 306: G147-G153, 2014.

8. Andreasen AS, Krabbe KS, Krogh-Madsen R, Taudorf S, Pedersen BK and Moller K: Human endotoxemia as a model of systemic inflammation. Curr Med Chem 15: 1697-1705, 2008.

9. Marshall JC, Walker PM, Foster DM, Harris D, Ribeiro M, Paice J, Romaschin AD and Derzko AN: Measurement of endotoxin activity in critically ill patients using whole blood neutrophil dependent chemiluminescence. Crit Care 6: 342-348, 2002.

10. Yoshioka N, Taniguchi Y, Yoshida A, Nakata K, Nishizawa T, Inagawa $\mathrm{H}$, Kohchi $\mathrm{C}$ and Soma $\mathrm{G}$ : Intestinal macrophages involved in the homeostasis of the intestine have the potential for responding to LPS. Anticancer Res 29: 4861-4865, 2009.

11. Cong X, Zhang Y, Yang NY, Li J, Ding C, Ding QW, Su YC, Mei M, Guo XH, Wu LL and Yu GY: Occludin is required for TRPV1-modulated paracellular permeability in the submandibular gland. J Cell Sci 126: 1109-1121, 2013.

12. Nolan JP: The role of intestinal endotoxin in liver injury: A long and evolving history. Hepatology 52: 1829-1835, 2010.

13. Trebicka J, Krag A, Gansweid S, Appenrodt B, Schiedermaier P, Sauerbruch T and Spengler U: Endotoxin and tumor necrosis factor-receptor levels in portal and hepatic vein of patients with alcoholic liver cirrhosis receiving elective transjugular intrahepatic portosystemic shunt. Eur J Gastroenterol Hepatol 23: 1218-1225, 2011.

14. Adachi Y, Moore LE, Bradford BU, Gao W and Thurman RG: Antibiotics prevent liver injury in rats following long-term exposure to ethanol. Gastroenterology 108: 218-224, 1995.

15. Diao H, Li X, Chen J, Luo Y, Chen X, Dong L, Wang C, Zhang C and Zhang J: Bletilla striata polysaccharide stimulates inducilble nitric oxide synthase and proinflammatory cytokine expression in macrophages. J Biosci Bioeng 105: 85-89, 2008.

16. Wang C, Sun J, Luo Y, Xue W, Diao H, Dong L, Chen J and Zhang J: A polysaccaride isolated from the medicinal herb Bletilla striata induces endothelial cells proliferation and vascular endothelial growth factor expression in vitro. Biotechnol Lett 28: 539-543, 2006

17. Wang Y, Liu J, Li Q, Wang Y and Wang C: Two natural glucomannan polymers, from Konjac and Bletilla, as bioactive materials for pharmaceutical applications. Biotechnol Lett 37: 1-8, 2015.

18. Luo Y, Diao H, Xia S, Dong L, Chen J and Zhang J: A physiologically active polysaccharide hydrogel promotes wound healing. J Biomed Mater Res A 94: 193-204, 2010.

19. Dong L, Xia S, Luo Y, Diao H, Zhang J, Chen J and Zhang J: Targeting delivery oligonucleotide into macrophages by cationic polysaccharide from Bletilla striata successfully inhibited the expression of TNF-alpha. J Control Release 134: 214-220, 2009.

20. Zhang Y, Lv T, Li M, Xue T, Liu H, Zhang W, Ding X and Zhuang Z: Anti-aging effect of polysaccharide from Bletilla striata on nematode Caenorhabditis elegans. Pharmacogn Mag 11: 449-454, 2015.

21. Wang Y, Liu D, Chen S, Wang Y, Jiang H and Yin H: A new glucomannan from Bletilla striata: Structural and anti-fibrosis effects. Fitoterapia 92: 72-78, 2014

22. Livak KJ and Schmittgen TD: Analysis of relative gene expression data using real-time quantitative PCR and the 2(-Delta Delta C(T)) method. Methods 25: 402-408, 2001.

23. Wang Y, Yang F, Xue J, Zhou X, Luo L, Ma Q, Chen YF, Zhang J, Zhang SL and Zhao L: Anti-schistosomiasis liverfibrosis effects of chlorogenic acid through IL-13/miR-21/Smad7 signaling interactions in vivo and in vitro. Antimicrob Agents Chemother 61: pii: e01347-16, 2017.

24. Tsochatzis EA, Bosch $\mathrm{J}$ and Burroughs AK: Liver cirrhosis. Lancet 383: 1749-1761, 2014.

25. Malaguarnera G, Giordano M, Nunnari G, Bertino G and Malaguarnera M: Gut microbiota in alcoholic liver disease: Pathogenetic role and therapeutic perspectives. World J Gastroenterol 20: 16639-16648, 2014.

26. Brun P, Castagliuolo I, Di Leo V, Buda A, Pinzani M, Palù G and Martines D: Increased intestinal permeability in obese mice: New evidence in the pathogenesis of nonalcoholic steatohepatitis. Am J Physiol 292: G518-G525, 2007.

27. Caine WR, Metzler-Zebeli BU, McFall M, Miller B, Ward TL, Kirkwood RN and Mosenthin R: Supplementation of diets for gestating sows with zinc amino acid complex and gastric intubation of suckling pigs with zinc-methionine on mineral status, intestinal morphology and bacterial translocation in lipopolysaccharide-challenged early-weaned pigs. Res Vet Sci 86: 453-462, 2009. 
28. Song HL, Lv S and Liu P: The roles of tumor necrosis factor- $\alpha$ in colon tight junction protein expression and intestinal mucosa structure in a mouse model of acute liver failure. BMC Gastroenterol 9: 70, 2009

29. Yang DH, Ye ZY, Jin B, He XJ, Zhang Q, Zhou WM, Xu WJ and Lu HX: Salvianolate inhibits cytokine gene expression in small intestine of cirrhotic rats. World J Gastroenterol 17: 1903-1909, 2011.

30. Shen L, Weber CR, Raleigh DR, Yu D and Turner JR: Tight junction pore and leak pathways: A dynamic duo. Annu Rev Physiol 73: 283-309, 2011.

31. Wang F, Graham WV, Wang Y, Witkowski ED, Schwarz BT and Turner JR: Interferon-gamma and tumor necrosis factor-alpha synergize to induce intestinal epithelial barrier dysfunction by up-regulating myosin light chain kinase expression. Am J Pathol 166: 409-419, 2005.

32. Muñoz L, José Borrero M, Ubeda M, Lario M, Díaz D, Francés R, Monserrat J, Pastor O, Aguado-Fraile E, Such J, et al: Interaction between intestinal dendritic cells and bacteria translocated from the gut in rats with cirrhosis. Hepatology 56: 1861-1869, 2012

33. Toda K, Kumagai N, Tsuchimoto K, Inagaki H, Suzuki T, Oishi T, Atsukawa K, Saito H, Morizane T, Hibi T and Ishii H: Induction of hepatic stellate cell proliferation by LPS-stimulated peripheral blood mononuclear cells from patients with liver cirrhosis. J Gastroenterol 35: 214-220, 2000.

34. Mitic LL, Van Itallie CM and Anderson JM: Molecular physiology and pathophysiology of tight junctions I. Tight junction structure and function: Lessons from mutant animals and proteins. Am J Physiol Gastrointest Liver Physiol 279: G250-G254, 2000.

35. Anderson JM and Van Itallie CM: Physiology and function of the tight junction. Cold Spring Harb Perspect Biol 1: a002584, 2009.

36. Gonzalez-Mariscal L, Betanzos A, Nava P and Jaramillo BE: Tight junction protein. Prog Biophys Mol Biol 81: 1-44, 2003.

37. Hu YJ, Wang YD, Tan FQ and Yang WX: Regulation of paracellular permeability: Factors and mechanisms. Mol Biol Rep 40 6123-6142, 2013

38. Furuse M, Hirase T, Itoh M, Nagafuchi A, Yonemura S, Tsukita S and Tsukita S: Occludin: A novel integral membrane protein localizing at tight junctions. J Cell Biol 123: 1777-1788, 1993.
39. Saitou M, Ando-Akatsuka Y, Itoh M, Furuse M, Inazawa J, Fujimoto K and Tsukita S: Mammalian occludin in epithelial cells: Its expression and subcellular distribution. Eur J Cell Biol 73: 222-231, 1997.

40. Li J, Ge R, Zhao C, Tang L, Li J and Li Q: Farrerol regulates occludin expression in hydrogen peroxide-induced EA.hy926 cells by modulating ERK1/2 activity. Eur J Pharmacol 734: 9-14, 2014.

41. Chen D, Li L, Yan J, Yang X, You Y, Zhou Y and Ling X: The loss of $\alpha$ SNAP downregulates the expression of occludin in the intestinal epithelial cell of acute pancreatitis model. Pancreatology 14: 347-355, 2014.

42. Lapierre LA: The molecular structure of the tight junction. Adv Drug Deliv Rev 41: 255-264, 2000.

43. Berkes J, Viswanathan VK, Sarkovic SD and Hecht G: Intestinal epithelial responses to enteric pathogens: Effects on the tight junction barrier, ion transport, and inflammation. Gut 52: 439-451, 2003.

44. Beltinger J, McKaig BC, Makh S, Stack WA, Hawkey CJ and Mahida YR: Human colonic subepithelial myofibroblasts modulate transepithelial resistance and sceretory response. Am J Physiol 277: C271-C279, 1999.

45. Stevenson BR, Siliciano JD, Mooseker MS and Goodenough DA Identification of ZO-1: A high molecular weight polypeptide associated with the tight junction (zonula occludens) in a variety of epithelia. J Cell Biol 103: 755-766, 1986.

46. Cario E, Gerken G and Podolsky DK: Toll-like receptor 2 enhances ZO-1-associated intestinal epithelial barrier integrity via protein kinase C. Gastroenterology 127: 224-238, 2004.

47. Chen J, Zhang R, Wang J, Yu P, Liu Q, Zeng D, Song H and Kuang Z: Protective effects of baicalin on LPS-induced injury in intestinal epithelial cells and intercellular tight junctions. Can J Physiol Pharmacol 93: 233-237, 2015. International (CC BY-NC-ND 4.0) License. 